University of Nebraska - Lincoln

DigitalCommons@University of Nebraska - Lincoln

\title{
Irradiation effects on microstructure change in nanocrystalline ceria - Phase, lattice stress, grain size and boundaries
}

\author{
P.D. Edmondson \\ University of Oxford, philip.edmondson@materials.ox.ac.uk
}

Y. Zhang

Oak Ridge National Laboratory, yanwen@utk.edu

S. Moll

Service de Recherches de Metallurgie Physique

F. Namavar

University of Nebraska Medical Center, fnamavar@unmc.edu

W.J. Weber

University of Tennessee - Knoxville, wjweber@utk.edu

Follow this and additional works at: https://digitalcommons.unl.edu/usdoepub

Edmondson, P.D.; Zhang, Y.; Moll, S.; Namavar, F.; and Weber, W.J., "Irradiation effects on microstructure change in nanocrystalline ceria - Phase, lattice stress, grain size and boundaries" (2012). US Department of Energy Publications. 357.

https://digitalcommons.unl.edu/usdoepub/357

This Article is brought to you for free and open access by the U.S. Department of Energy at DigitalCommons@University of Nebraska - Lincoln. It has been accepted for inclusion in US Department of Energy Publications by an authorized administrator of DigitalCommons@University of Nebraska - Lincoln. 


\title{
Irradiation effects on microstructure change in nanocrystalline ceria - Phase, lattice stress, grain size and boundaries
}

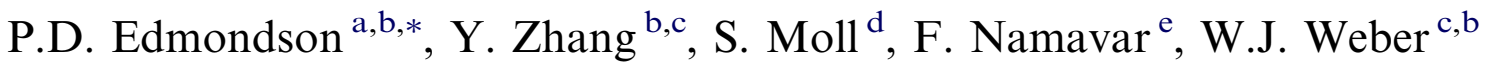 \\ ${ }^{a}$ Department of Materials, University of Oxford, Parks Road, Oxford OX1 3PH, UK \\ ${ }^{\mathrm{b}}$ Materials Science and Technology Division, Oak Ridge National Laboratory, Oak Ridge, TN 37831, USA \\ ${ }^{\mathrm{c}}$ Department of Materials Science and Engineering, University of Tennessee, Knoxville, TN 37996, USA \\ ${ }^{\mathrm{d}}$ CEA-DEN, Service de Recherches de Métallurgie Physique, Centre d'Études de Saclay, 91191 Gif-sur-Yvette Cedex, France \\ ${ }^{\mathrm{e}}$ University of Nebraska Medical Center, Omaha, NB 68198, USA
}

Received 2 March 2012; received in revised form 2 July 2012; accepted 4 July 2012

Available online 10 August 2012

\begin{abstract}
With a wide variety of applications in numerous industries, ranging from biomedical to nuclear, ceramics such as ceria are key engineering materials. It is possible to significantly alter the materials functionality and therefore its applications by reducing the grain size to the nanometer size regime, at which point the unique varieties of grain boundaries and associated interfaces begin to dominate the material properties. Nanocrystalline films of cubic ceria deposited onto Si substrates have been irradiated with $3 \mathrm{MeV} \mathrm{Au}^{+}$ions at temperatures of 300 and $400 \mathrm{~K}$ to evaluate their response to irradiation. It was observed that the films remained phase stable. Following a slight stress relief stage at low damage levels, the overall lattice is extremely stable up to high irradiation dose of $\sim 34$ displacements per atom. The grains were also observed to undergo a temperature-dependent grain growth process upon ion irradiation. This is attributed to a defect-driven mechanism in which the diffusion of defects from the collision cascade is critical. Formation of dislocations that terminate and stabilize at symmetric grain boundaries may be the limiting factor in the grain growth and overall energy reduction of the system. Utilizing ion modification, possible improvement of the adhesion of thin films and reduction of the probability of detrimental effects of stress-induced problems are discussed.
\end{abstract}

(C) 2012 Acta Materialia Inc. Published by Elsevier Ltd. All rights reserved.

Keywords: Nanocrystalline ceria; Ion irradiation; Lattice effects; Dislocations

\section{Introduction}

Ceramics are important engineering materials, with practical applications in numerous industries. In the automotive industry, ceramics may be used in catalytic convertor technologies, as oxygen sensors and as high-performance brake discs. The binary oxide ceramics such as $\mathrm{HfO}_{2}, \mathrm{ZrO}_{2}$ and $\mathrm{CeO}_{2}$ are also being explored as potential high- $k$ dielectrics [1-4] in the electronics industry to enable continuous reduction in scaling of devices. Ceramics are also heavily utilized in the nuclear industry, with $\mathrm{UO}_{2}$ and $\mathrm{PuO}_{2}$ being used as

\footnotetext{
* Corresponding author at: Department of Materials, University of Oxford, Parks Road, Oxford OX1 3PH, UK.

E-mail address: philip.edmondson@materials.ox.ac.uk (P.D. Edmondson).
}

fuel materials, $\mathrm{SiC}$ as a component in TRISO and QUADRISO fuel pellets, and $\mathrm{ZrO}_{2}$ and $\mathrm{CeO}_{2}$ as possible components in inert matrix fuels, such as rock-like oxide [5] or cermet $[6,7]$ fuels, or as part of high-level nuclear waste forms $[8,9]$. Ceramics may also be used as hydrophilic, highwear-resistant coatings for replacement joints in biomedical applications [10].

The ceramic ceria $\left(\mathrm{CeO}_{2}\right)$ has potential uses in many of the applications described above. Due to its exceptional mixed electronic-ionic conduction, $\mathrm{CeO}_{2}$ is widely used in solid oxide fuel cells. $\mathrm{CeO}_{2}$ has a cubic fluorite structure from room temperature up to its melting point of $\sim 3000 \mathrm{~K}$, and is isostructural in nature with $\mathrm{UO}_{2}$ and $\mathrm{PuO}_{2}$ [11]. $\mathrm{CeO}_{2}$ exhibits similar irradiation defect behaviour as $\mathrm{UO}_{2}$ and $\mathrm{PuO}_{2}$ [11], and it is therefore often used as a non-radioactive surrogate in studies of nuclear fuel systems in which fuel 
systems are typically composed of micron-sized (or greater) grains of the fuel material, e.g. $\mathrm{UO}_{2}$. However, by reducing the grain size of the material to the nanosized regime, it is possible to tailor the materials properties [12-15]. Nanostructured ceramic materials may provide improved operational fuel and cladding performance and better response in loss-of-coolant accidents in terms of enhanced radiation resistance, improved mechanical strength, reduced cracking and higher thermal conductivity due to the high density of interfaces and grain boundaries (GBs).

GBs are two-dimensional defects, and can be approximately characterized as low $\sum$ boundaries, general GBs or misorientated GBs [16]. Here, $\sum$ refers to the reciprocal density of the coincident site in accordance with the coincident site lattice (CSL) model [17]. Thus, by definition, CSL GBs are symmetric defects in that neighbouring grains with a common GB are exact mirror images about the GB, as are low-angle GBs. Misorientated GBs do not show any symmetry of neighbouring grains, and are thus asymmetric in nature.

Low $\sum$ GBs may exhibit interesting mechanical and kinetic properties such as high resistance to sliding, fracture and corrosion [17]. In the context of nuclear fuels, GBs may heavily influence the material properties that govern fuel performance. In particular, fission gases such as Xe and $\mathrm{Kr}$, which have low solid solubility in materials [18], may migrate to the GBs and agglomerate, resulting in the nucleation of bubbles, which may be detrimental to fuel performance and structural integrity. Interface structure and GB property may play important roles in bubble formation and subsequent release of fission gases.

As biomedical coatings, ceramics are deposited onto a biocompatible substrate (e.g. Ti) in high-friction/wear regions (e.g. ball joints). The requirements for this application are different from those of the nuclear applications discussed above. The material must be extremely resistant to wear, biologically inert and hydrophilic [10]. Typically, thin films of the ceramic are deposited onto the substrate. One technique that is used for thin-film deposition for biomedical applications is that of ion-beam-assisted deposition (IBAD) [10]. In this technique, low-energy ions $(\leqslant 1500 \mathrm{eV})$ are used to effectively stitch engineered nanocrystals to the substrate. This technique is advantageous and results in the formation of ultrahydrophilic surfaces via the Lotus effect, enhancement of film adhesion [10] and reduction of the stress in the film by eliminating stress-related problems such as buckling, microcracking and peeling. The adhesion of the films can be improved by in situ removal of monolayers of contaminants prior to deposition, increasing the reactivity of the substrate/coating atoms, generating a nanoscopically rough surface at the interface due to ion beam mixing, increasing nucleation density, enhancing surface mobility of the coating atoms or decreasing the formation of interfacial voids, or a combination of the above [10]. IBAD may also be successfully utilized to control the surface morphology, density, stress level, crystallinity, grain size and orientation, and chemical composition.
In the work presented here, thin films of nanocrystalline ceria have been irradiated with heavy ions in order to evaluate the response of the nanocrystalline ceria to radiation damage for nuclear applications and the possibility of using ion beams to engineer the thin films for other applications, such as biomedical.

\section{Experimental methods}

Thin films of approximately $330 \mathrm{~nm}$ thick, nanostructured $\mathrm{CeO}_{2}$ (NSC) were grown on (001) silicon substrates with an $\sim 5 \mathrm{~nm}$ buffer layer of $\mathrm{SiO}_{2}$ using an ion-beamassisted deposition system (Mill Lane engineering, Lowell, MA) at the Nanotechnology Laboratory at the University of Nebraska Medical Center [10]. The thin films were then irradiated with $3.0 \mathrm{MeV} \mathrm{Au}{ }^{+}$ions up to fluence of $6.4 \times 10^{15}$ ions cm $\mathrm{cm}^{-2}$ at temperatures of 300 and $400 \mathrm{~K}$. The ion flux was kept constant during the irradiation (approximately $10^{11}$ ions $\mathrm{cm}^{-2} \mathrm{~s}^{-1}$ to reduce beam heating effects on the sample) and the beam was rastered over the surface to ensure a uniform irradiation. The ion beam energy was chosen such that the energy deposited into the film was maximized whilst minimizing the Au concentration in the thin film. The corresponding displacements per atom (dpa) values were calculated using SRIM 2008.01 full-cascade simulations. The parameters used for the simulations were a sample density of $\sim 6.3 \mathrm{~g} \mathrm{~cm}^{-3}$, with threshold displacement energies of 27 and $56 \mathrm{eV}$ for the $\mathrm{O}$ and $\mathrm{Ce}$ atoms respectively [19,20]. This gave an average fluence-to-dpa conversion factor of $0.54 \mathrm{dpa}$ per $10^{14}$ ions $\mathrm{cm}^{-2}$ [21].

Post-irradiation characterization was performed using the complementary techniques of Rutherford backscattering spectroscopy (RBS), transmission electron microscopy (TEM) and glancing incidence X-ray diffraction (GIXRD). Both the ion irradiation and ion beam analysis was performed using the $3.0 \mathrm{MV}$ tandem accelerator facilities in the Environmental Molecular Science Laboratory (EMSL), located at Pacific Northwest National Laboratory (PNNL). Characterization of the elemental composition was performed using RBS in random orientation utilizing a $2.0 \mathrm{MeV} \mathrm{He}^{+}$beam with two $\mathrm{Si}$ detectors located at scattering angles of $150^{\circ}$ and $165^{\circ}$ relative to the incoming ion beam.

Cross-sectional TEM specimens were prepared by mechanically polishing down to a thickness of $\sim 15$ $20 \mu \mathrm{m}$ using a tripod polishing technique. In order to achieve electron transparency, the mechanical polishing is followed by ion milling in a Gatan precision ion polishing system with a beam energy of $4.5 \mathrm{keV}$, gradually decreased to $3 \mathrm{keV}$. The specimens were evaluated using a JEOL 2010 transmission electron microscope operating at $200 \mathrm{keV}$.

The GIXRD measurements were recorded on a Philips X'Pert MPD diffractometer operating at $45 \mathrm{kV}$ and $40 \mathrm{~mA}$ using a fixed $\mathrm{Cu}$ anode with an $\mathrm{X}$-ray wavelength of $1.54187 \AA$. A Göbel mirror for the incident beam and a 0.27 radian parallel plate collimator for the diffracted 
beam were used in this study. The glancing incident angle was fixed at $\omega=5^{\circ}$ to avoid strong diffraction intensities from the underlying Si substrate. Analysis of the XRD patterns was conducted using the commercial software JADE from Materials Data, Inc. and the PDF4+ database from ICSD. The nanocrystalline sizes were all determined from the main diffraction peaks using pseudo-Voigt profiles, and are in good agreement with the grain sizes observed in the TEM data.

\section{Results}

\subsection{As-deposited film}

A TEM micrograph of the as-deposited NSC film is shown in Fig. 1a. The NSC film is approximately $330 \mathrm{~nm}$ thick and has a random texture. Above the $\mathrm{Si}$ substrate is a $5 \mathrm{~nm}$ buffer layer of $\mathrm{SiO}_{2}$, on top of which is the NSC film. The high-resolution image (Fig. 1b) shows that the $\mathrm{Si}$ substrate is highly crystalline, the $\mathrm{SiO}_{2}$ buffer layer is amorphous and the NSC film consists of nanocrystalline grains that are randomly orientated. The random orientation is confirmed by the selected area electron diffraction (SAED) pattern in Fig. 1c, which is indicative of polycrystalline material. Also shown are the crystallographic indices for the cubic fluorite structure, indicating that the NSC film is of the cubic phase. The GIXRD results shown in Fig. 2 confirm that the as-deposited film is of the cubic fluorite structure, and also reveal that the average grain size of the film was $\sim 6 \mathrm{~nm}$. The GIXRD peaks show that the (311) and (222) peaks become more distinct and individually resolvable with increasing ion dose, a phenomenon
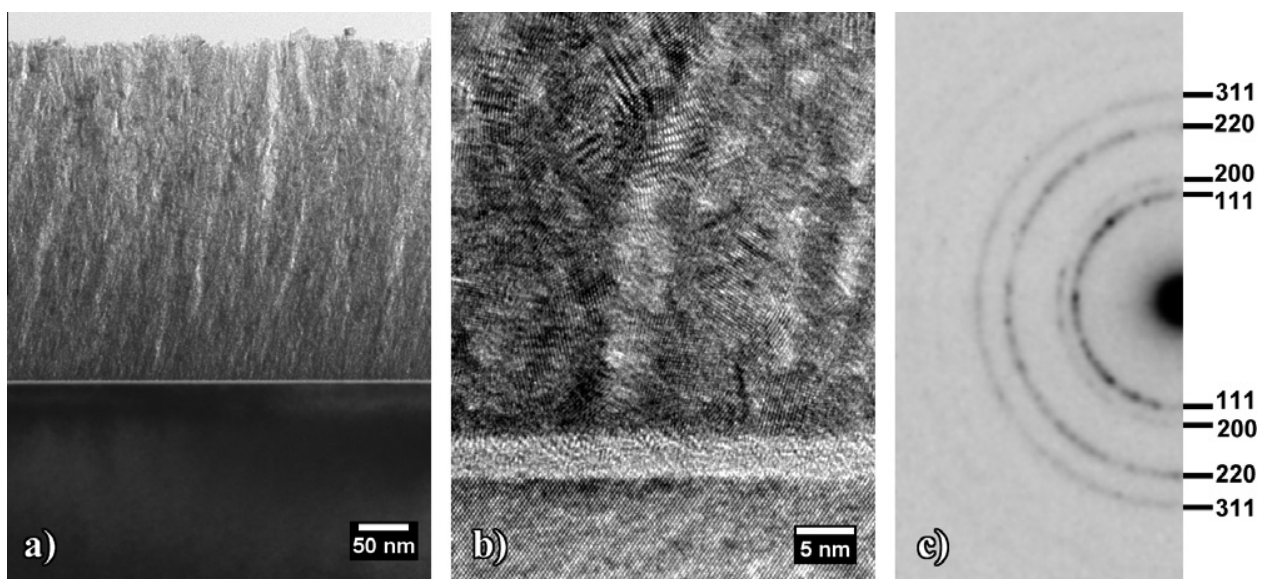

Fig. 1. TEM micrographs of the NSC film at (a) low magnification, diffraction contrast; (b) high-resolution phase contrast; and (c) SAED pattern from the NSC film, with crystallographic indices labelled.
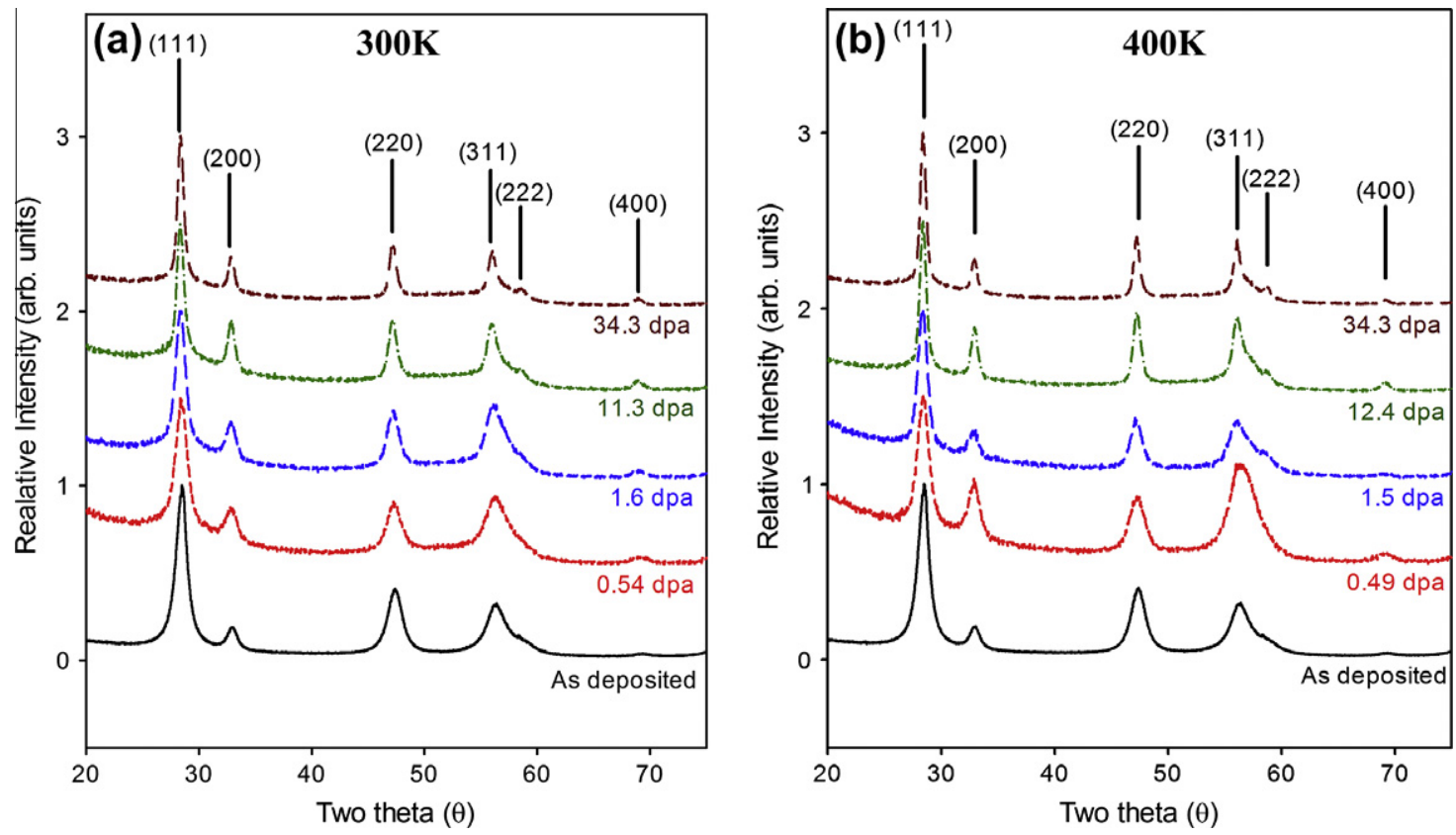

Fig. 2. GIXRD results of the NSC films irradiated with $3 \mathrm{MeV} \mathrm{Au}^{+}$to doses of up to $\sim 34 \mathrm{dpa}$ at (a) $300 \mathrm{~K}$ and (b) $400 \mathrm{~K}$. The results show that the cubic phase is retained regardless of the irradiation dose and temperature, whilst the narrowing of the diffraction peaks is indicative of grain growth. 

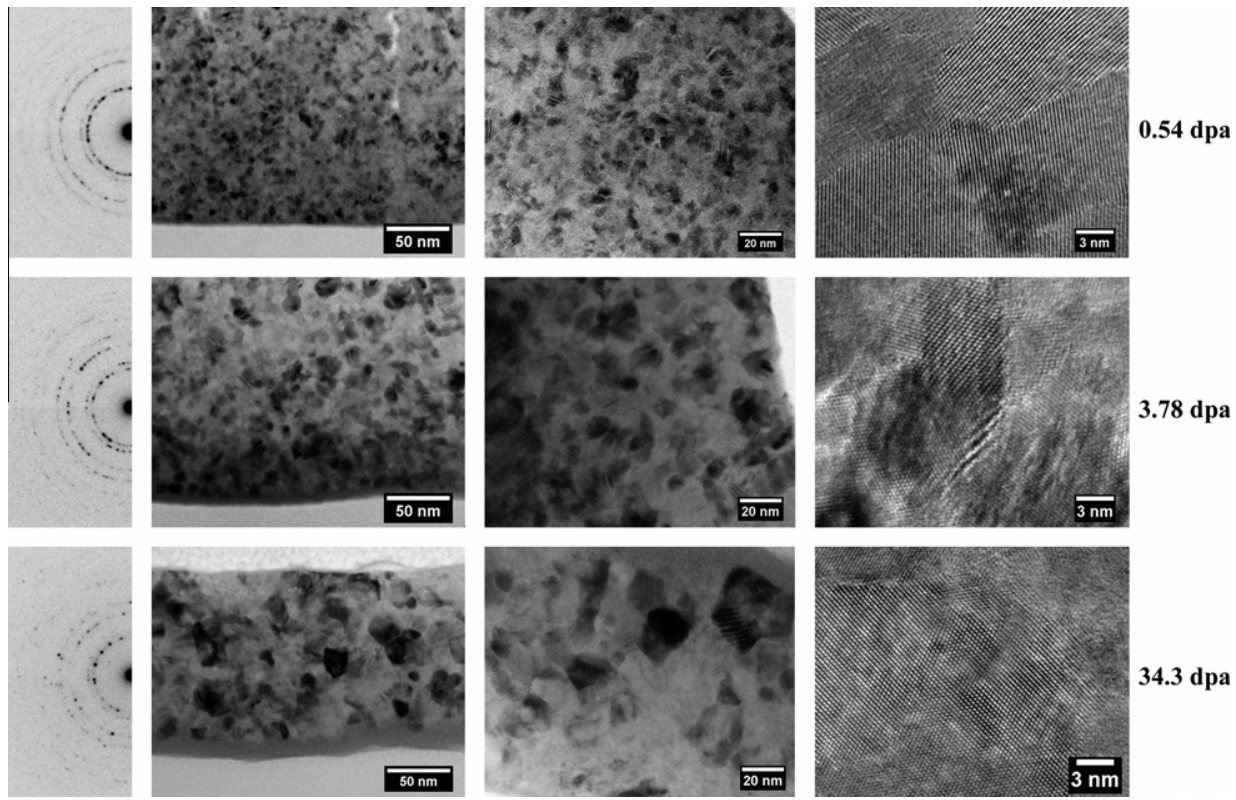

Fig. 3. Series of SAED patterns, low-magnification, high-magnification and high-resolution TEM micrographs of the NSC films irradiated at $300 \mathrm{~K}$ to various doses (indicated on the right). Grain growth is evident from the SAED pattern and the low-magnification images. High-quality grain boundaries are also apparent. The underlying Si substrate is rendered amorphous at the lowest ion dose.

consistent with grain growth (this will be discussed further in Section 4.2). RBS reveals that the $\mathrm{O} / \mathrm{Ce}$ ratio was $\sim 2$, indicating that the film is stoichiometric.

\subsection{Irradiated films}

\subsection{1. $300 \mathrm{~K}$ irradiation}

SAED patterns, bright-field diffraction contrast images and high-resolution phase contrast images for the samples irradiated to $0.54,3.78$ and $34.3 \mathrm{dpa}$ at a temperature of $300 \mathrm{~K}$ are shown in Fig. 3. The SAED patterns show that, as the dose is increased up to $\sim 34 \mathrm{dpa}$, a refinement of the patterns occurs. This refinement is observed as a discretization of the diffraction spots. Such an observation is indicative of grain coarsening. Moreover, the bright-field diffraction contrast images indicate that the nanocrystalline grains undergo growth, with the grains that were initially approximately $6 \mathrm{~nm}$ in diameter increasing to $\sim 17 \mathrm{~nm}$. This
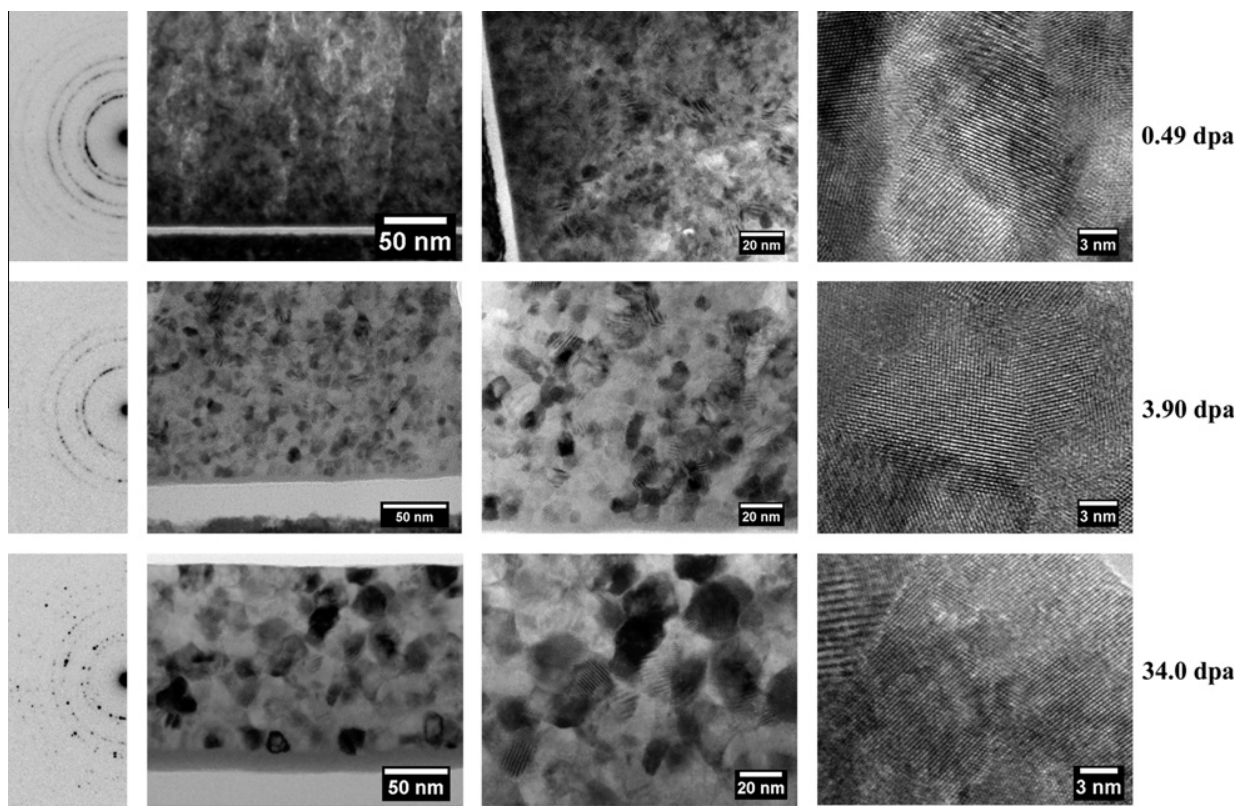

Fig. 4. Series of SAED patterns, low-magnification, high-magnification and high-resolution TEM micrographs for NSC films irradiated at $400 \mathrm{~K}$ to various doses (indicated on the right). As with the $300 \mathrm{~K}$ results, grain growth is evident, as are high-quality grain boundaries. Unlike the $300 \mathrm{~K}$ irradiation, amorphization of the substrate is somewhat retarded and grows with increasing ion dose. 
grain-coarsening effect is also evident in the GIXRD results shown in Fig. 2 by the narrowing of the diffraction peaks. Examination of the diffraction contrast and phase contrast TEM images also reveal that no visible three-dimensional voids or vacancy/interstitial clusters were formed in the film. The high-resolution phase contrast images also reveal that the nanocrystalline grains are of apparent high quality and that the grain boundaries are sharp. RBS analysis of the chemical composition in the film revealed that no loss of oxygen from the film occurred as has been observed in similar materials (nanocrystalline zirconia) $[12,15]$.

\subsection{2. $400 \mathrm{~K}$ irradiation}

SAED patterns, bright-field diffraction contrast and high-resolution phase contrast TEM images recorded for samples irradiated to $0.49,3.90$ and $34.0 \mathrm{dpa}$ at a temperature of $400 \mathrm{~K}$ are shown in Fig. 4. Similar to the $300 \mathrm{~K}$ irradiated sample, the SAED patterns show discretization of the diffraction spots indicative of grain growth. The diffraction contrast TEM images confirm grain growth, similar to the GIXRD results in Fig. 2. The high-resolution TEM images reveal that the nanocrystalline grains and grain boundaries are again of high quality regardless of the irradiation dose. Again, no three-dimensional defects were observed within the detection limit. RBS analysis further demonstrated that no loss of oxygen occurred.

\section{Discussion}

\subsection{Phase and lattice stability}

From the GIXRD results and the SAED patterns shown in Figs. 2-4, it is evident that the cubic fluorite phase of the film is maintained during the irradiation. This is inferred by the absence of the generation or disappearance of diffraction peaks. It can be seen in both the 300 and $400 \mathrm{~K}$ GIXRD results that the (222) reflection is not clearly observed in the as-deposited and low dpa samples. As the dpa increases to 1.5 and above, the (222) reflection becomes visible. This is not due to the generation of the (222) reflection but, rather, is a result of the irradiation-induced grain coarsening causing a refinement (narrowing) of the diffraction peaks. As the diffraction peaks become narrower, the (222) and (311) reflections become more distinct and resolvable.

Several phases of ceria are known to exist, and depend heavily on the oxygen content of the material [22]. The cubic fluorite phase exists for $\mathrm{CeO}_{2-x}$ when $0<x<0.5$, and the hexagonal phase forms when $x \geqslant 0.5$, i.e. $\mathrm{Ce}_{2} \mathrm{O}_{3}$. Reports have been made about the existence of the rhombohedral phase for $\mathrm{CeO}_{1.82}$, although subsequent studies have been unable to confirm the existence of this phase [22]. It therefore seems likely that only the cubic fluorite and hexagonal phases may exist at atmospheric pressures and temperatures.

During ion irradiation, ion-solid interactions occur that result in the permanent displacement of some atoms from their lattice sites. With increasing ion fluence, this generally results in the generation of saturation concentrations for interstitials and vacancies, as observed previously for alpha-irradiated $\mathrm{CeO}_{2}$.[11] Thus, it is expected that irradiation of the thin NSC film should result in a saturation level of defects that will be visible in the GIXRD results due to the distortion of the lattice.

Measurements of the lattice parameter as a function of dose and grain size, using the (111) reflection from the GIXRD data, are shown in Fig. 5. It is evident that, at low dose levels, there is a dramatic reduction in the lattice parameter, followed by stabilization (Fig. 5a). This suggests that there is an initial stress relief of the film at low dose levels, after which the lattice appears to stabilize, despite the introduction of significant damage. The graph displaying the lattice parameter as a function of grain size shows a similar effect (Fig. 5b), in which an initial rapid decrease in lattice parameter is observed, followed by stabilization. The initial decrease is most likely associated with stress relief of the as-deposited film rather than any grain size dependence. However, Deshpande et al. [23] have previously shown that, for grains of $\mathrm{CeO}_{2}$ in the size range of interest here $(7-20 \mathrm{~nm})$, there is a significant variation in the lattice parameter $(\sim 0.005 \mathrm{~nm})$. This is in contradiction to the results presented here, whereby, over a similar size range, a shift of only $\sim 0.001 \mathrm{~nm}$ lattice variation is observed. Whilst Deshpande et al. attributed this to the generation of oxygen
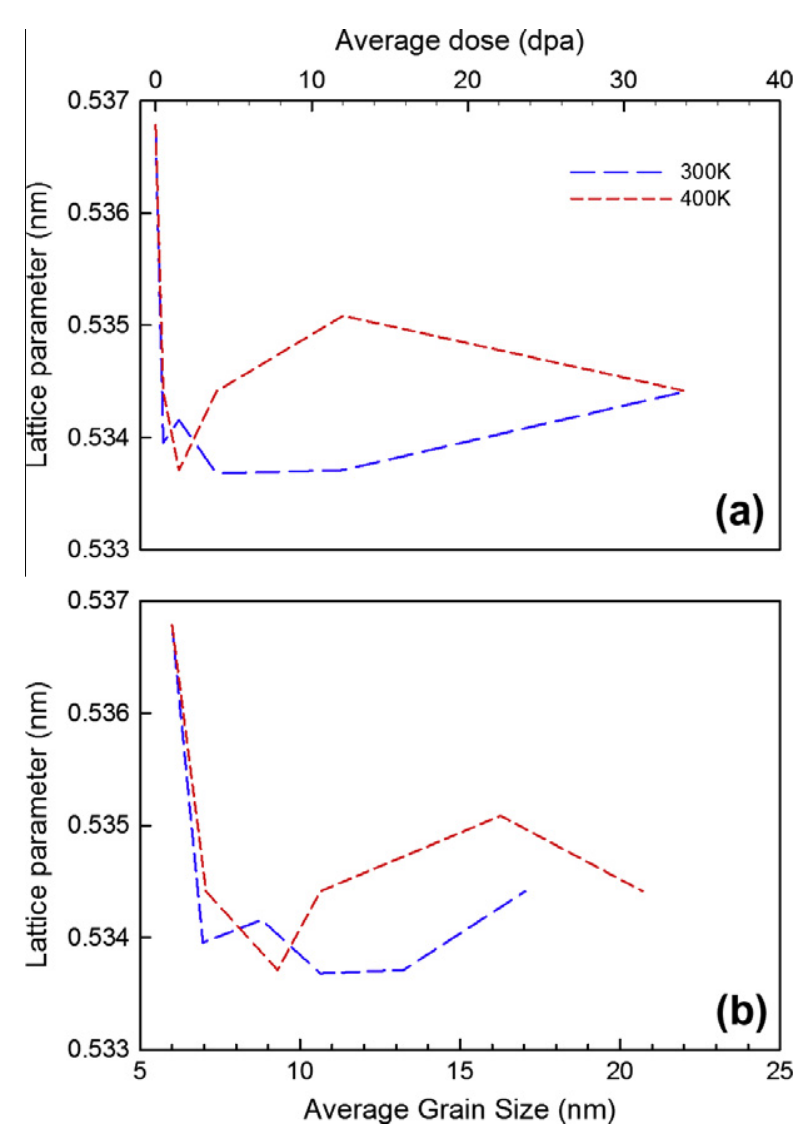

Fig. 5. Graphs showing the variation of lattice parameter, as measured using GIXRD, of the NSC film during irradiation as a function of (a) ion dose and (b) average grain size. 
vacancies within the grains [23] (introduced during the synthesis process), the samples in that study consisted of free-standing grains, where the surface area-to-volume effects may not be negligible.

The slight lattice variation observed in this work is in contrast to the increase in lattice parameter observed previously $(0.7 \%)$ in alpha-irradiated sintered $\mathrm{CeO}_{2}$ [11], as well as to that observed during similar irradiations of nanocrystalline zirconia thin films in which a significant shift in the lattice parameter was measured [24], the extent of which was found to be dependent on the irradiation temperature. That observed shift in the zirconia work was attributed to the formation and loss of oxygen interstitials, subsequently leading to the accumulation of an excess of oxygen vacancies during the ion irradiation, with different charge states depending on the irradiation temperature. The results presented here on ceria films show that the films undergo minor lattice distortion $(<0.2 \%)$, suggesting limited accumulation of defects due to effective recombination and annihilation processes.

These observations may be explained as follows. Once the initial relaxation phase is complete (at very low doses), the change in lattice parameter with increasing dose is a direct result of the competition between recombination processes in the grains and defect loss/migration to the grain boundaries, which are dependent on the grain size. Based on previous results [11], the effective recombination radius for irradiation-induced defects in sintered (large grain) $\mathrm{CeO}_{2}$ at $300 \mathrm{~K}$ is estimated to be about $2.5 \mathrm{~nm}$ due to high oxygen vacancy mobility. This is comparable to the initial grain size; thus, recombination and defect loss (primarily highly mobile oxygen vacancies and less mobile oxygen interstitials) to the accessible grain boundaries are expected to dominate during irradiation at $300 \mathrm{~K}$ to low doses, leaving the grain interiors relatively defect free with no lattice expansion. As the grains grow in size with increasing dose, the defect diffusion distances to grain boundaries for the less mobile oxygen interstitials [11] become too large and some excess interstitials accumulate in the grain interiors, leading to the observed lattice expansion at grains sizes exceeding about $12 \mathrm{~nm}$ diameter. Below this grain size, any defect produced within the grain interior is within the recombination distance of either a grain boundary or other defects within the grain interiors. This process also helps to retard the grain growth, as will be discussed below. In the case of irradiation at $400 \mathrm{~K}$, the much higher mobility of the vacancies leads to more enhanced vacancy loss to the grain boundaries and less recombination, leaving an excess of interstitials in the grain interiors at lower doses (smaller grain size). The concentration of excess interstitials increases with ion dose and grain size, leading to the observed increase in lattice parameter. Eventually, the higher interstitial concentration at $400 \mathrm{~K}$ leads to clustering, which results in the observed decrease in lattice parameter. Measurements of the (111) fringe spacing by high-resolution TEM (HREM; Fig. 6) indicates that there is little variation in the lattice, suggesting that the

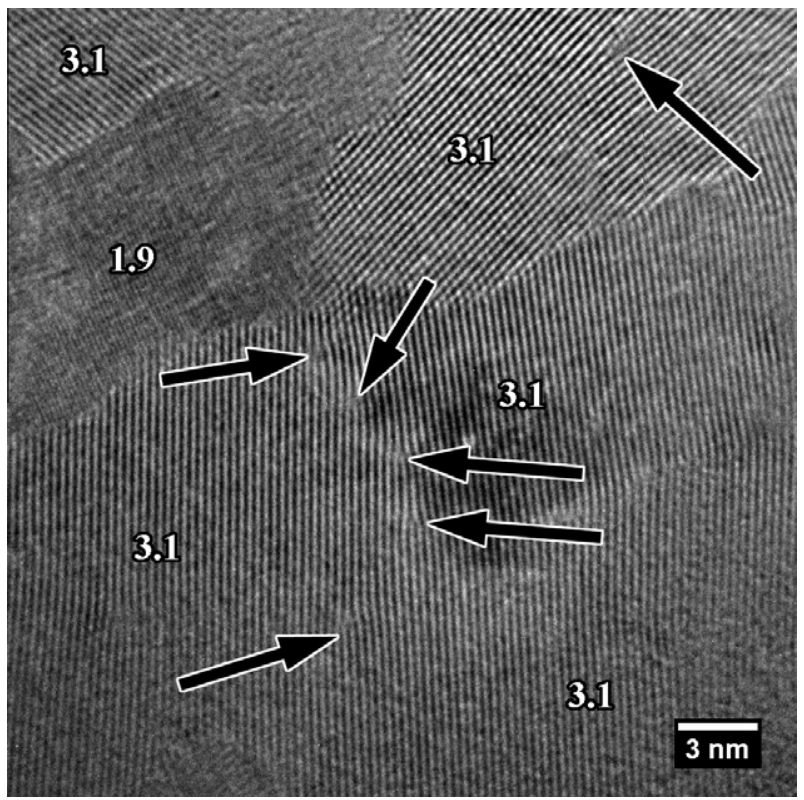

Fig. 6. HREM micrograph of the NSC film irradiated to $0.54 \mathrm{dpa}$ at $300 \mathrm{~K}$. High-quality grain boundaries are visible, as is a single dislocation terminating in the upper-rightmost grain. Overlaid are the lattice spacings measured from the individual grains. The $3.1 \AA$ and $1.9 \AA$ measured spacings relate to the $d_{111}$ and $d_{220}$ lattice planes, respectively. Arrows indicate the presence of dislocations that terminate at grain boundaries.

defect accumulation is not as significant as in previous similar experiments on zirconia [24].

\subsection{Grain growth and grain boundary evolution}

During irradiation of the NSC films, the grains undergo growth, as seen in Figs. 3 and 4. The dependence of grain size on dose is summarized in Fig. 7, where it can easily be seen that the grains undergo a rapid growth at low dpa levels before beginning to stabilize somewhat at higher dpa levels. Indeed, increasing the dose further (to a value of $108 \mathrm{dpa}$ ) results in additional growth of the grain size to an asymptotic value of $\sim 25 \mathrm{~nm}$ [21]. Previous work has shown that grain growth of nanocrystalline metallic systems under irradiation [25] follows the equation $D^{n}-D_{0}^{n}=k \Phi t$, where $n=3, D$ is the grain size for a given dose, $D_{0}$ is the initial grain size, $K$ is a constant and $\Phi t$ is the ion dose. For nanocrystalline ceramic films, it has been shown that the exponent, $n$, does not equal $3[15,21]$; nor does it equal 2 - a value assigned for thermal growth of nanocrystalline metallic grains. This suggests that there is a different grain growth mechanism occurring in nanocrystalline ceramics (ceria and zirconia). It has been proposed that a defect-stimulated grain growth mechanism is the dominant process in nanocrystalline ceramics under irradiation $[15,21]$, with the diffusion of the defects to the grain boundaries playing a vital role - as discussed above. While it would normally be expected that grain growth should not occur in ceramics at these temperatures, the nanocrystalline morphology of the samples, coupled with the potential for a non-equilibrium concentration of defects 


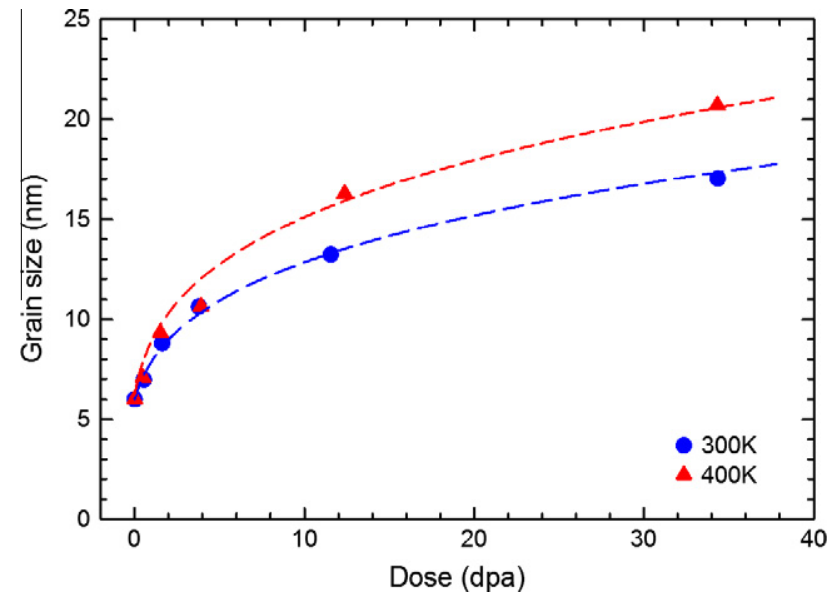

Fig. 7. Graph showing the grain growth as a function of ion dose for the films irradiated with $3 \mathrm{MeV} \mathrm{Au}{ }^{+}$at substrate temperatures of 300 and $400 \mathrm{~K}$. Lines are added to guide the eye.

that may be present in the sample due to the synthesis process, could result in thermally induced grain growth. However, an as-deposited nanocrystalline ceria film was annealed at $400 \mathrm{~K}$ for $5 \mathrm{~h}$, during which time no grain growth was observed, demonstrating that thermal growth at $400 \mathrm{~K}$ is negligible [26]. However, it is not clear what the driving force behind this defect-stimulated grain growth mechanism (i.e. the defects responsible for the growth) is, or the net gradient under which growth occurs (i.e. to what extent the grains grow). In an attempt to elucidate this second question, the evolution of the grain boundaries has been studied as a function of ion dose.

Electron backscattering diffraction (EBSD) and orientation imaging microscopy (OIM) are techniques typically used to study grain boundaries to obtain information about the misorientation angles and coincident lattice information. However, the nanocrystalline nature of the grains in this study means that their grain size is below the resolution limit of these techniques, so in this work the symmetry of the grain boundaries is studied using HREM.

A typical HREM image of the NSC film is shown in Fig. 6. The majority of the lattice fringes observed result from the (1 11 ) planes (fringe spacing $3.1 \AA$ ). Occasionally, lattice fringes from the (220) planes are observed (fringe spacing $1.9 \AA$ ). Furthermore, the grain boundaries are not necessarily symmetric. Here the grain boundary is defined as being symmetric when two adjacent grains of similar orientation, i.e. displaying similar lattice spacings, are symmetric along the grain boundary. Examples of symmetric and asymmetric grain boundaries are shown in Fig. 8.

The percentages of measured symmetric and asymmetric grain boundaries as a function of ion dose are shown in Fig. 9. Initially, approximately all of the grain boundaries are asymmetric. As the dose increases, there is a tendency for the grain boundaries to become symmetric prior to saturating at doses of $\sim 10 \mathrm{dpa}$ and above. The rate at which saturation occurs is dependent on the irradiation temperature, with the $300 \mathrm{~K}$ irradiation reaching saturation quicker than
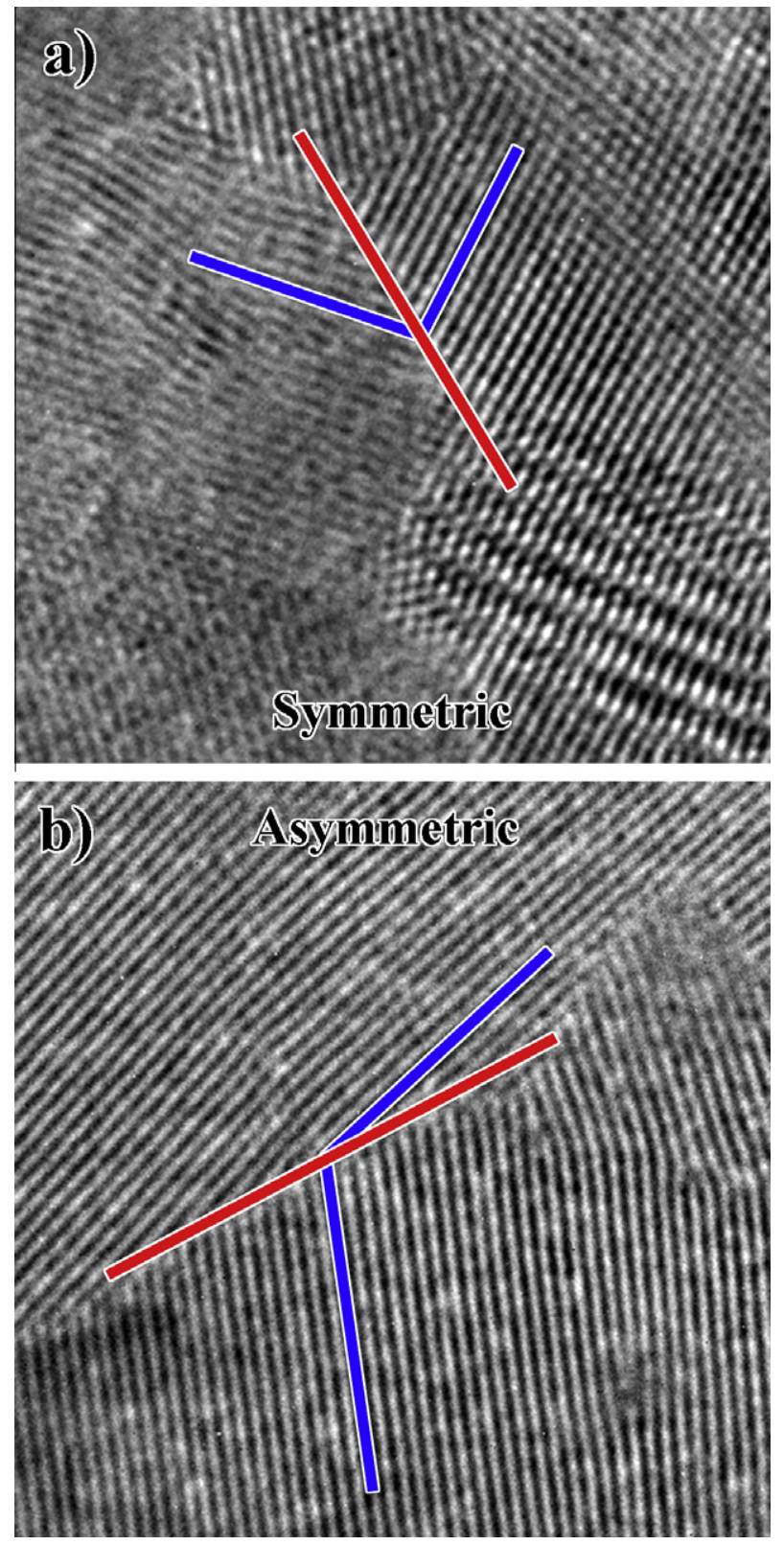

Fig. 8. TEM micrographs showing examples of symmetric (a) and asymmetric (b) grain boundaries.

the $400 \mathrm{~K}$ irradiation. The change in grain boundary state, from asymmetric to symmetric, may be attributed to a reduction in the energy level of the system. In polycrystalline ceramics, an electrical potential is formed across asymmetric grain boundaries due to the breaking of the local atomic structure, leading to the formation of dipoles at the boundary [17]. During the ion implantation, the resultant displacement damage can cause random bond breaking and energy transfer to the electronic structure, leading to localized electronic excitations and charges at defects and interfaces, which may rupture or change the nature of the covalent and ionic bonds. This is of particular relevance in the ceria system, the cation structure of which is unique in that it is characterized by bonds bridging across the interface to anions [17]. Thus, the displacement damage produced 


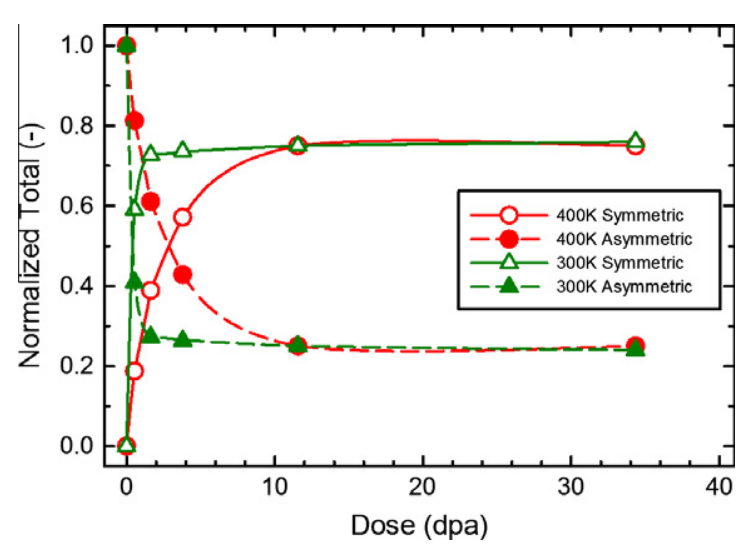

Fig. 9. Graph showing the symmetric-asymmetric relationships between grain boundaries during irradiation of the NSC film. It is apparent that the grain boundaries tend to become symmetric during the irradiation via an energy minimization mechanism. Lines are added to guide the eye.

during the irradiation may facilitate the atomic reordering at the grain boundary interface, reducing the local potential. This leads to an overall lowering of the energy level of the system (more stable) when the grain boundaries become more symmetric. It is therefore energetically more favourable for the grain boundaries to become more symmetric during the irradiation to minimize the system energy by reducing the electric fields across the grain boundaries.

Typically, the dominant CSL for materials having similar structures to $\mathrm{CeO}_{2}$, i.e. $\mathrm{UO}_{2}$, is the $\sum 9$, having a symmetry angle [16] of $38.94^{\circ}$; however, this has also been determined in materials with significantly larger grain sizes. Moreover, as mentioned above, it is not possible to use techniques such as EBSD or OIM to determine the CSL values in such fine-grained materials as those in this study. Additionally, the high degree of curvature observed in these nanoscale grains will add to further inaccuracies in the measurement of the angles between grains and thus in assignment of CSL values that are typically derived from planar interfaces [27]. As such, it may only be speculated that the dominant CSL is that of the $\sum 9$ in this study.

Previous studies of the grain boundaries in $\mathrm{UO}_{2}[16,17]$ have shown that Schottky defects exist at the high-misorientation (asymmetric) grain boundaries, helping to stabilize the system by reducing the energy at the grain boundary. The lower angle grain boundaries and CSL (symmetric) grain boundaries are stabilized by dislocations terminating at the grain boundaries $[16,17]$. Some examples of dislocation termination are shown in Fig. 6.

It is clear from Fig. 9 that there is a tendency for asymmetric GBs to become symmetric during ion irradiation, and that there is a temperature dependency on this transition. The results suggest that the transition between asymmetric and symmetric GBs must be interlinked with the transition of Schottky defects and dislocations being the dominant defects at the GBs. Moreover, the results suggest that the asymmetric GBs are high-misorientation GBs, and the symmetric GBs are dominated by low-angle CSL structures.
As has been discussed, the transition of the GBs from asymmetric to symmetric is accompanied by the transition from Schottky defects to dislocations being the dominant defects at the GBs. This transition occurs at a faster rate for the $300 \mathrm{~K}$ irradiation in comparison to that at $400 \mathrm{~K}$. This is attributed to the growth of dislocations during the irradiation at $400 \mathrm{~K}$. It is well known that dislocations readily form in ion-irradiated ceria. In experiments on single crystal $\mathrm{CeO}_{2}$, it was observed that at lower irradiation temperatures higher densities of smaller dislocations were formed, whereas a lower density of larger dislocations was produced at higher temperatures [28]. This change in dislocation size at different temperatures was attributed to the $\mathrm{Ce}$ vacancy mobility. However, the migration energy of the $\mathrm{Ce}$ vacancy has been estimated experimentally to be $2.3 \mathrm{eV}$ [11] and calculated to be $5.3 \mathrm{eV}$ [19]. Based on these activation energies and the observation [11] that the Ce vacancy is essential immobile below $675 \mathrm{~K}$, it seems improbable that the Ce vacancy is mobile at the temperatures in either this study or the work of Ye et al. [28]. On the other hand, the oxygen vacancy in $\mathrm{CeO}_{2}$ has a measured and calculated migration energy of $0.52 \mathrm{eV}$ [19], and the migration energy of the oxygen interstitial has been experimentally estimated to be $1.8 \mathrm{eV}$ [11] and calculated to be $1.13 \mathrm{eV}$ [19]. Consequently, the oxygen vacancy is likely to be highly mobile at both 300 and $400 \mathrm{~K}$, given its low migration energy, whereas the oxygen interstitial will be somewhat more mobile at $400 \mathrm{~K}$ compared to $300 \mathrm{~K}$. Accordingly, if the oxygen interstitial is an important defect in the growth of dislocations, it can be expected that a lower density of larger dislocations will form during the higher temperature irradiation, and a high density of small dislocations will be produced at the lower temperature irradiation. These higher densities of dislocations will rapid meet the GBs and effectively pin the grain boundaries in the symmetric state. The lower dislocation densities formed at higher temperatures will result in a reduced number of dislocations encountering a grain boundary, thus the transition between unpinned asymmetric GBs and pinned symmetric GBs will occur over a longer dose range window.

This has a significant impact on the methods by which highly structurally stable thin films of nanocrystalline ceria are formed. In this study, the material was prepared using an ion-beam-assisted deposition technique [10] in which an $\mathrm{Ar}^{+}$beam is utilized during the deposition process to aid in the reduction of free energy of the system and therefore to reduce the probability of the film undergoing buckling or microcracking. These results clearly indicate that, despite the use of low-energy Ar irradiation to relieve stress, there is still a reasonably high amount of stress within the film post-deposition. It is possible that further reduction of stress could be accomplished by refinement of the parameters used in the ion-beam-assisted deposition technique itself, or through the use of a post-deposition structural modification by low-dose high-energy ion irradiation, which may be attainable at the industrial scale through 
the use of existing technology currently employed for electronic device production.

\section{Conclusions}

Thin films of nanocrystalline ceria grown on top of silicon substrates have been irradiated with $3 \mathrm{MeV} \mathrm{Au}^{+}$ions at 300 and $400 \mathrm{~K}$. It has been demonstrated that the phase of the material is extremely stable during the irradiation, whereas the lattice undergoes relaxation upon low-dose irradiation. The phase and structural stability under high-dose irradiation indicate that the nanocrystalline ceria films are extremely irradiation tolerant. The grains are observed to undergo a temperature-dependent, defect-stimulated growth. Rearrangement of the symmetry of the grain boundaries from initially asymmetric to symmetric under irradiation is attributed to a lowering of the system energy by an atomic rearrangement at the grain boundary, leading to a reduction in the electric potential at the grain boundaries.

\section{Acknowledgements}

This work was supported as part of the Materials Science of Actinides, an Energy Frontier Research Center funded by the U.S. Department of Energy, Office of Science, Office of Basic Energy Sciences. A portion of the research was performed at the Environmental Molecular Sciences Laboratory (EMSL), a national user facility sponsored by the Department of Energy's Office of Biological and Environmental Research and located at Pacific Northwest National Laboratory (PNNL). Part of this research performed using the Shared Research Equipment (SHaRE) User Facility at Oak Ridge National Laboratory, which is sponsored by the Office of Basic Energy Sciences, U.S. Department of Energy.

\section{References}

[1] Chi D, McIntyre PC. Appl Phys Lett 2004;85:4699.

[2] King PJ, Werner M, Chalker PR, Jones AC, Aspinall HC, Basca J, et al. Thin Solid Films 2011;519:4192.
[3] Thompson DP, Dickins AM, Thorp JS. J Mater Sci 1992;27: 2267.

[4] Wilk GD, Wallace RM, Anthony JM. J Appl Phys 2000;87:484.

[5] Nitani N, Kuramoto K, Yamashita T, Nihei Y, Kimura Y. J Nucl Mater 2003;319:102.

[6] Fernández A, Konings RJM, Somers J. J Nucl Mater 2003;319: 44.

[7] Pouchon MA, Nakamura M, Hellwig C, Ingold F, Degueldre C. J Nucl Mater 2003;319:37.

[8] Smith KL, Colella M, Cooper R, Vance ER. J Nucl Mater 2003;321:19.

[9] Weber WJ, Navrotsky A, Stefanovsky S, Vance ER, Vernaz E. MRS Bull 2009;34:46.

[10] Namavar F, Cheung CL, Sabirianov RF, Mei W-N, Zeng XC, Wang G, et al. Nano Lett 2008;8:988.

[11] Weber WJ. Radiat Eff 1984;83:145.

[12] Edmondson PD, Zhang Y, Namavar F, Wang CM, Zhu Z, Weber WJ. Nucl Instrum Methods Phys Res Sect B: Beam Interact Mater Atoms 2011;269:126.

[13] Kovalenko MV, Scheele M, Talapin DV. Science 2009;324:1417.

[14] Norris DJ, Efros AL, Erwin SC. Science 2008;319:1776.

[15] Zhang Y, Jiang W, Wang C, Namavar F, Edmondson PD, Zhu Z, et al. Phys Rev B 2010;82:184105.

[16] Nerikar PV, Rudman K, Desai TG, Byler D, Unal C, McClellan KJ, et al. J Am Ceram Soc 2011;94:1893.

[17] Nerikar P, Stanek CR, Phillpot SR, Sinnott SB, Uberuaga BP. Phys Rev B 2010;81:064111.

[18] Donnelly SE. Radiat Eff Defects Solids 1985;90:1.

[19] Guglielmetti A, Chartier A, Brutzel LV, Crocombette J-P, Yasuda K, Meis C, et al. Nucl Instrum Methods Phys Res Sect B: Beam Interact Mater Atoms 2008;266:5120.

[20] Yasunaga K, Yasuda K, Matsumura S, Sonoda T. Nucl Instrum Methods Phys Res Sect B: Beam Interact Mater Atoms 2008;266:2877.

[21] Zhang Y, Edmondson PD, Varga T, Moll S, Namavar F, Lan C, et al. Phys Chem Chem Phys 2011;13:11946.

[22] Trovarelli A. Catal Rev 1996;38:439.

[23] Deshpande S, Patil S, Kuchibhatla SVNT, Seal S. Appl Phys Lett 2005;87:133113.

[24] Edmondson PD, Weber WJ, Namavar F, Zhang Y. Scripta Mater 2011;65:675.

[25] Kaoumi D, Motta AT, Birtcher RC. J Appl Phys 2008;104: 073525.

[26] Edmondson PD, Zhang Y, Moll S, Varga T, Namavar F, Weber WJ. Phys Rev B 2012;85:214113.

[27] Brokman A, Balluffi RW. Acta Metall 1981;29:1703.

[28] Ye B, Kirk MA, Chen W, Oaks A, Rest J, Yacout A, et al. J Nucl Mater 2011;414:251. 